\title{
1 \\ The Relationship Between Genotype and Phenotype: Some Basic Concepts
}

\author{
Philip Stanier and Gudrun Moore
}

\section{Introduction}

Without even considering early fetal loss, it is reported that as many as $3.5 \%$ of all live-born babies have some kind of major abnormality, referred to as a birth defect. Actual incidences may vary according to locality, culture, ethnicity and the efficiency of recognition and reporting. If minor abnormalities such as cleft lip are included, then the incidence is nearer to $5 \%$. In the Western world, birth defects constitute the greatest single cause of infant mortality and have a major impact on national health care budgets (http://www.modimes.org/).

In this introductory chapter some basic precepts and concepts are presented and explained. For a comprehensive introduction to embryonic development per se, the reader is referred to any one of several excellent publications that already exist (e.g. Alberts et al., 2002; Gilbert, 2003; Wolpert, 2002). What this chapter attempts to provide is the information that might be necessary for a clinician or advanced student specializing in paediatric medicine to understand and appreciate in context what follows. In that sense, an element of unorthodoxy might be discerned by some readers. However, we hope that this rationale will be justified as the reader progresses through the book.

Embryos, Genes and Birth Defects, Second Edition Edited by Patrizia Ferretti, Andrew Copp, Cheryll Tickle and Gudrun Moore (C) 2006 John Wiley \& Sons, Ltd 


\section{The relationship between genotype and phenotype}

The term 'genotype' is generally used to refer to the genetic make-up or constitution of an individual organism, be it virus, fruit fly or human. In contrast, we use the word 'phenotype' to cover the form and functioning of an individual, to the extent that it may encompass metabolism and behaviour (and thus we can refer to 'behavioural phenotypes'). The word 'genotype' is subtly but distinctly different from the term 'genome', which refers not to the totality of genes in an individual cell but to the array of genes in a complete haploid set of genes characteristic for that species. In this sense, a genome is a species-specific concept, whereas genotype is a concept applying to an individual of the species in question.

The complexity of the phenotype reflects largely but not entirely the complexity of the genotype. However, there is not necessarily a simple and direct relationship, since genome size and genome complexity are rather different entities. Overall genome size, in terms of DNA, is to some extent determined by the relative proportion of nonprotein coding sequences contained within it. Thus, some plant, insect and amphibian species contain far more total DNA in their genomes than does Homo sapiens, even though they are phenotypically simpler and contain fewer genes (indeed, some amphibian species contain up to $9 \times 10^{11}$ nucleotide bases per haploid genome, as opposed to the $2.85 \times 10^{9}$ nucleotides recently sequenced in humans; International Human Genome Sequencing Consortium, 2004). Much of this increase in DNA content is thought to represent a greater than normal proportion of non-coding, repetitive sequences. If we consider genome complexity in terms of the number of genes present, then a more systematic relationship emerges. In simple organisms, such as viruses, the limited number of genes in the genome can be accurately determined. However, for more complex multicellular organisms, total gene number is an estimate based on confirmed genes and potential coding regions identified by predictive methods. Therefore, the size of these estimates has changed as our ability to visualize the DNA sequence and our understanding of genomic organization has evolved. Currently, Drosophila melanogaster, the fruit fly, is estimated to contain some 14000 genes in its genome, whereas the genome of Homo sapiens is thought to comprise between 20000 and 25000 . However, this latter set of figures is still subject to revision and does not take into account the considerable protein variation that can accrue from alternate usage and splicing of exons or the existence of functional noncoding RNAs.

Whereas gene mapping refers to identification of the chromosomal location of an individual gene, genome mapping is a programme of research designed to identify the chromosomal location of all genes in the genome of a particular species. Although it is the international Human Genome Project that has received wide media attention, it should be noted that genome mapping projects for other species are also under way or recently completed. These include a number of model organisms, such as the mouse, fruitfly, toad and nematode worm, as well as those of economically important food species, such as cow, pig and chicken (http://www.ncbi.nlm.nih.gov/Genomes/ index.html). The mapping of individual genes, or of candidate gene loci, means that 


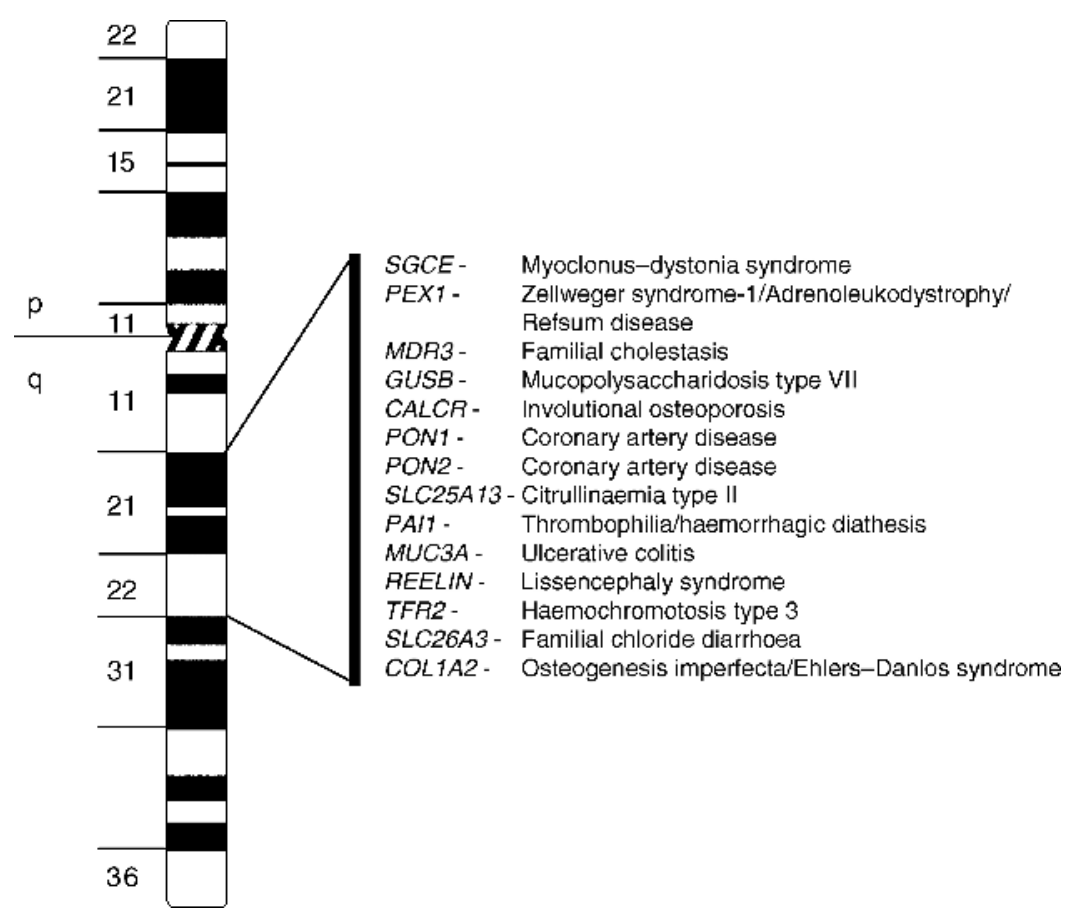

Figure 1.1 Congenital malformation with gene mutations mapping to 7q21-q22. More than 1700 have been identified throughout the genome, including $>80$ on chromosome 7 (see http:// www.ncbi.nlm.nih.gov/LocusLink/ and Chapter 2)

chromosomal 'maps' of congenital abnormality can be drawn up (see Chapters 2, 3 and 4; also OMIM: http://www.ncbi.nlm.nih.gov/entrez/query.fcgi?db=OMIM), whereby the location of genes, in which mutation produces a particular dysmorphology or inherited metabolic disease, can be displayed (Figure 1.1).

At this point we should ask ourselves what kind of information is encoded within the genes. Are the genes really the 'blueprint' to which they are often analogized? A blueprint implies some kind of descriptive specification. Is that indeed how the genome is organized? In fact, the information content of genes is one-dimensionally complex, since it is specified by the nature of the linear sequence of nucleotide bases along the DNA molecule. In dramatic contrast, the phenotype is three-dimensionally complex (and four-dimensionally complex if we include dynamic phenomena, such as metabolism and homeostasis, rather than just morphology); yet the linear nucleotide sequence itself conveys no sense of what the phenotype might look like. To appreciate just how phenotypic complexity might be generated we have to move away from the rather dated analogy of a descriptive specification and think of the genome and its implementation as a generative programme. The more appropriate and meaningful analogy of origami has been proposed to illustrate the characteristics 
of a generative programme (Wolpert, 1991). Here, the instructions for creating a topologically complex shape from a sheet of paper contain within them no description of the final outcome. The complexity is generated progressively by implementing those instructions, which may in themselves be very simple, even though the outcome is complex. In this way, the genome, or at least the developmentally significant parts of it, can be seen as assembly rules for building an embryo.

In one sense, genes 'simply' encode proteins. Transcription of a gene produces a message that is translated from the four-letter alphabet (nucleotides) of the nucleic acids to the approximately 20-letter alphabet (amino acids) of the proteins, by virtue of the genetic code. The primary structure of a protein, i.e. the linear sequence of amino acids, together with any post-translational modifications, determines its secondary and tertiary structure. Proteins endow cells with properties such as characteristic metabolisms, behaviour, polarity, adhesiveness and receptivity to signals (Figure 1.2) and it is this functional level that marks the implementation of those assembly rules. Within the increasingly multicellular embryo, cell interactions and inductions are initiated, cell lineages are established, and morphogenesis, growth and histogenesis proceed. Thus, interactions of proteins, cells and tissues during

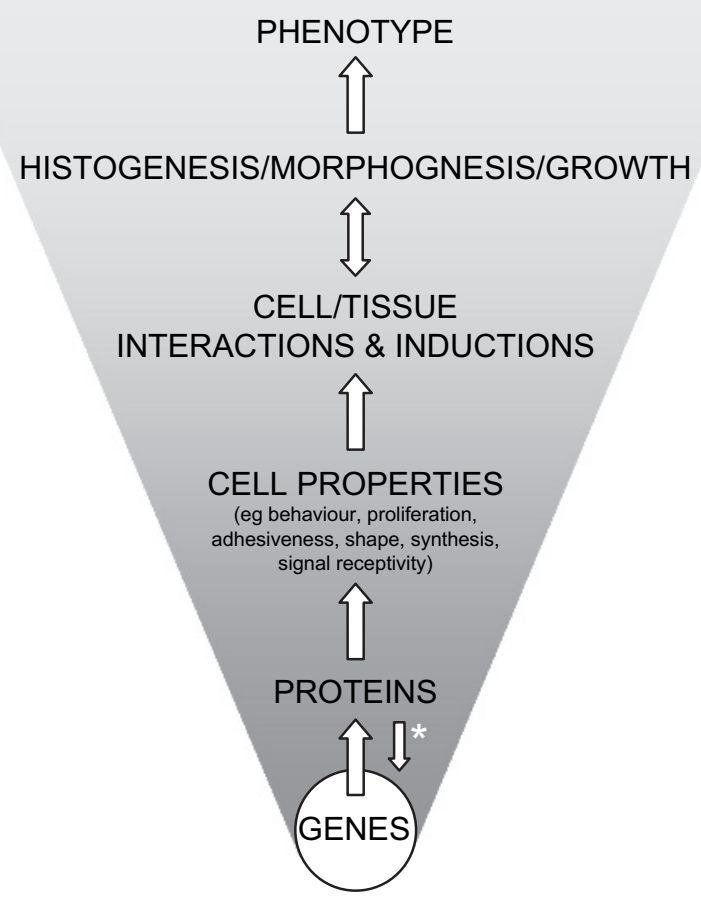

Figure 1.2 Causal relationship between genotype and phenotype. Higher-order complexity is generated progressively by the interaction of proteins, of cells and of tissues during development. The asterisked 'return' arrow between genes and proteins represents the controlling role on gene expression of transcription factors encoded by regulatory genes 
development generate progressively higher-order complexity (Figure 1.2), from the one-dimensional complexity of the genotype and primary protein structure to the three-dimensionally complex phenotype. Embryonic development is therefore a typical generative programme. From a limited range of fundamental cell properties, an almost infinite range of complex phenotypes can be built, simply by deploying these cell properties in varying ways. The diverse range of phenotypic from across extant and extinct species bears witness to the morphogenetic power of these basic cell properties over an evolutionary time-scale.

Thus, it is the morphogenetic potential of cell properties and the mechanisms of embryonic development that causally link genotype and phenotype. And from this brief and perhaps simplistic rationalization, one can see that during development there will be significant, higher-order events taking place in the absence of direct genetic control but which are themselves the inevitable consequences of genetic specification (Figure 1.2, from the level of 'cell properties' upwards). Thus, the phenotypic expression of an individual's genotype is influenced by a variety of nongenetic factors that might involve variables such as diet, infection and ageing. These factors can have direct effects on gene expression or may influence more subtle control mechanisms, such as DNA or histone methylation. This class of phenomena is sometimes described as epigenetic and, clearly, much morphological complexity is generated within this so-called epigenetic domain (Alberch, 1982; McLachlan, 1986; Gottesman and Hanson, 2005).

Developmental biologists are interested in defining assembly rules and elucidating their operation at tissue, cellular and molecular/genetic levels. To understand dysmorphogenesis it is necessary to clarify what happens when certain assembly rules are either mis-specified or wrongly interpreted and a birth defect results. Clearly, understanding a particular birth defect involves much more than simply identifying a mutated gene or an environmental teratogen. It requires knowledge of the consequences of these on the mechanisms operating within the embryo, an understanding of how the generative programme has been perturbed and how that produces an abnormal phenotype. Furthermore, just as an understanding of normal development can help clarify abnormal development, so analysis of abnormal development can sometimes throw light on hitherto unknown aspects of normal mechanisms.

Before leaving this topic, it should be noted that in Figure 1.2 there is feedback indicated from proteins to genes (see reverse arrow). This reflects the fact that the role of some proteins is to bind to DNA, typically in a highly sequence-specific manner. Genes that encode such proteins are referred to as 'regulatory genes' and the proteins themselves known as 'transcription factors', since they control (either upregulate or downregulate) transcriptional activity of the gene to which they have bound. In essence, genes work in hierarchies, with regulatory genes controlling the expression of 'downstream' genes and with elements of 'cross-talk' between regulatory genes themselves. The definition of such genetic cascades and signalling pathways is a very topical issue in contemporary developmental biology and this is reflected by the prominence given to it by many of the contributors to this volume. 
Such genes are, of course, pivotally important in the normal life of the cell, in its synthetic and metabolic activity, homeostasis and proliferation, but during embryonic development they have multiple and crucial roles in determining cell fate. Although many of the genes identified to date as being involved in birth defects encode enzymes or structural proteins, it is not surprising that numerous families of regulatory genes have been established as playing important roles in dysmorphogenesis (see later).

Having discussed some aspects of the genotype-phenotype relationship, it is now appropriate to point out that it can be simplistic to always interpret dysmorphogenesis on the basis of a 'one gene:one (dysmorphic) phenotype' model. It is clear that, in some cases, a diversity of phenotypes can emerge from mutations in a single gene, each disease or dysmorphic phenotype reflecting a different mutation within that gene. Thus, different mutations in the receptor tyrosine kinase gene, $R E T$, can result in familial medullary thyroid carcinoma, multiple endocrine neoplasia types $2 \mathrm{~A}$ and 2B (all of which accords with its original recognition as an oncogene) and in Hirschsprung's disease, a developmental anomaly of the gut (reviewed by Manié et al., 2001; and see Chapter 11). This last disorder appears to be the consequence of a failure of RET-expressing neural crest cells to migrate normally and establish a parasympathetic innervation to the gut. The thyroid cancer-associated syndromes all result from mutations causing specific amino acid substitutions that apparently alter the functionality of the receptor tyrosine kinase encoded by RET (i.e. gain-offunction mutations that may lead to hyperplasia of the RET-expressing tissues). In contrast, the Hirschsprung mutations comprise deletion, insertion, frameshift, nonsense and missense mutations that lead to a loss of function. The phenotype can be explained as due to haploinsufficiency, whereby a threshold sensitivity to absence of $50 \%$ of the gene product (due to a mutated allele) is sufficient to perturb the development of the cells normally expressing that particular gene. In this case, it is the neural crest progenitors of the gut parasympathetic neurones that are affected, leaving other RET-expressing cell populations in the embryo apparently unscathed, due to tissue-specific differences in the threshold sensitivity (Manie et al., 2001). Interestingly, RET mutations that affect one of four extracytoplasmic cysteine residues have been found in Hirschsprung's patients, as well as patients with MEN2A and familial medullary thyroid carcinoma. These findings have raised the idea that a single mutation has opposing effects, depending on the tissue in which RET is expressed, and results in uncontrolled proliferation in endocrine cell types and apoptosis in enteric neurons (reviewed in Manié et al., 2001). Furthermore, mutations in RET are found only in about half of the familial cases of Hirschsprung's disease and then frequently with variable penetrance. This suggests a higher level of complexity, involving the interaction of other genes or non-coding variants, often referred to as modifiers. Co-inheritance of mutations in distinct loci but with additive effect gives rise to a multi- or polygenic inheritance model. In this case, each of the individual mutations alone may be considered risk factors, as they are insufficient to cause the phenotype alone but do so when inherited together. 
The causality of birth defects is not necessarily genetic in origin and various aetiological categories can be recognized:

- Chromosomal anomalies (e.g. trisomies, translocations)

- Polygenic disorders

- Single gene mutations

- Environmental/teratogenic factors

- Multifactorial aetiology

- Unknown aetiology

Each of these six categories presents its own set of problems in determining how a particular birth defect is generated (see Chapters 2, 3, 4 and 6). It might be argued that events occurring within the epigenetic domain referred to earlier can be extended to environmental influences on development. The embryo does not occupy a completely protected and privileged environment and, in some respects, is as open to effects from its environment as the neonate, juvenile or adult. Indeed, the recognition that the intrauterine experience of the fetus is strongly influenced by maternal nutritional or hormonal status is pivotal in determining later susceptibility to a number of adult diseases, such as diabetes and coronary heart disease (reviewed by Barker, 1995).

Clearly, the phenotype, be it adult or embryonic, is always the product of the combined effects of genetic and environmental influences (Sykes, 1993), but the relative contributions of each can differ for each aspect of the phenotype (Figure 1.3). Thus, Down's syndrome, as a trisomy disorder, reflects a condition that is $100 \%$ genetic, whereas a neural tube defect such as spina bifida (see Chapter 8) may have a

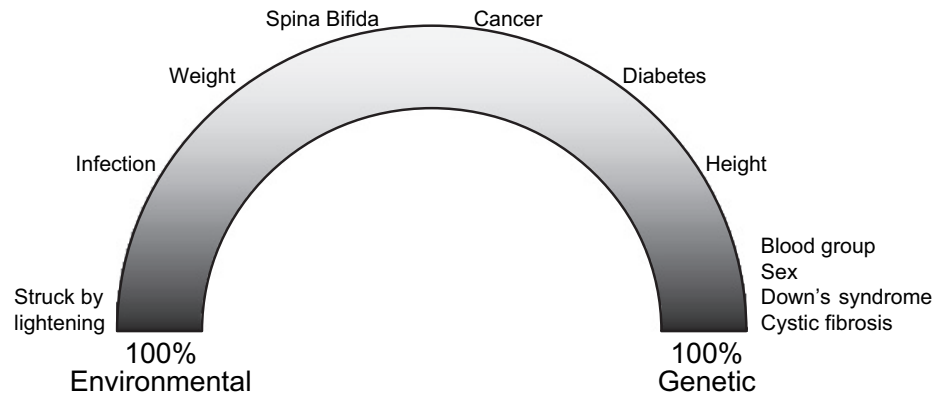

Figure 1.3 Interplay between environmental and genetic factors in the determination of phenotype. The relative importance of each will vary according to the particular phenotype, or aspect of phenotype, under consideration (after Sykes, 1993) 
strong environmental component in its aetiology, coupled with a possible genetic predisposition in some cases (reviewed by Marsh, 1994).

Even though the majority of birth defects have a genetic component, the extent of interaction between genotype and environment is still poorly understood and, in research studies, often neglected. Thus, the majority of animal studies assessing the teratogenicity and reproductive toxicity of environmental factors have frequently failed to take into account the different genotypes of the various strains of animal species used (discussed by Copp, 1994). Yet we are increasingly aware of human genes, which increase susceptibility to environmental teratogens. Examples of this include common polymorphisms that affect either protein levels or the activity of enzymes that metabolize the teratogens resulting from cigarette smoking, alcohol or drug intake (Polifka and Friedman, 2002). We may conclude that, in elucidating the complex relationship between genotype and embryonic phenotype, whether it be in the context of normal development or dysmorphogenesis, environmental factors may sometimes be critical (see Chapter 6).

\section{The role of 'model' systems}

To understand the mechanisms of development inevitably means dismantling and/or perturbing the embryo in some way. Very little has ever been learnt of mechanisms by simply observing embryonic development. Traditionally, developmental biologists dismantle and reassemble embryos, or parts of embryos, at the level of gene, cell, tissue or organ. In this way we learn how the system responds to perturbation, and through that we can elucidate the functional role of the component parts, sometimes down to the level of an individual nucleotide base within a DNA codon. For example, a change in a single nucleotide in the bicoid gene of Drosophila will actually reverse the anteroposterior axis of the embryo (Fronhöfer and Nusslein-Volhard, 1986; Struhl et al., 1989).

The ability to manipulate DNA in the laboratory has brought an unparalleled precision and finesse to developmental analysis, bringing exquisite control to generescue, knock-out, overexpression, ectopic expression and regulatory element studies. Thus, transgenic technology (see Chapter 5) can be considered as the most sophisticated of strategies, following in the great tradition of experimental perturbation started in the nineteenth century with the emergence of experimental embryology, epitomized by the German Entwicklungsmechanik ('developmental mechanics') school established by Wilhelm Roux and colleagues. However, it is important to comment that molecular biology as it exists now has not rendered traditional experimental embryology redundant. The molecular biology monoculture that some feared 15-20 years ago has not prevailed and what we see emerging today, and which is well reflected in the following chapters, is a pragmatism in which molecular approaches are creatively integrated with cellular and tissue approaches. For instance, a well-designed 'cut 'n' paste' tissue grafting experiment can generate results with profound implications at the molecular level (see e.g. some of the grafting 
experiments described in Chapter 7), and can itself direct further analysis at the molecular level.

This theme of perturbational analysis to reveal mechanisms means that the human embryo is not a system of choice, at least not after the 14-day limit set by the regulating authorities in Britain, and manifest in the Human Fertilization and Embryology Act, 1990 (and see Burn and Strachan, 1995; Table 1.1). Most dysmorphogenesis is likely to have its inception during the major stages of morphogenesis and organogenesis, starting with neurulation during the fourth week in the human embryo (Larsen, 2001). Disruptions earlier than that are likely to result in spontaneous abortion and be lost; indeed, it has been estimated that at least $15 \%$ of all human pregnancies end as spontaneous abortions after implantation (Warburton and Fraser, 1964).

Nevertheless, human fetal tissues are being used in biomedical research, particularly in the context of somatic gene therapy, fetal cell transplantation, haematopoietic stem cell transplantation and fetal organ transplantation (reviewed by Reed et al., 1995). However, many research programmes almost always use developmentally late fetal material, which is of little if any use in studying embryonic expression of genes implicated in birth defects. For this specific purpose, human embryo banks have been established (reviewed by Burn and Strachan, 1995), using material obtained from terminations and collected with full ethical approval. Reports on the expression of genes causally involved in dysmorphogenesis are now commonplace (see Chapter 2) and the use of such data in the long-term development of preventive and therapeutic clinical strategies is likely to escalate over the next few years.

However, in order to study developmental mechanisms during the crucial stages of morphogenesis and organogenesis we are, of necessity, obliged to use animal model systems. In Chapters 7-17 you will find reference to work using embryonic systems as diverse as zebrafish, Xenopus, chick and mouse. Are we to view these simply as models or research surrogates for the human embryo (see discussion by Monk, 1994)? In fact, developmental research is often driven by reasons of scholarship, and animal models are more typically studied for their own intrinsic interest, in the context of comparative biology and evolution (Bard, 1993). Nevertheless, several essential concepts that have significantly enhanced our understanding of human dysmorphology have emerged from analysis of animal model systems; the developmental field concept, as applied to dysmorphogenesis (Opitz, 1985), and chromosomal imprinting (Harwell imprinting site plus Otago site for human imprinted genes; Monk, 1994; see Chapter 2) are two obvious examples. However, when animal model systems are seen as 'research surrogates' for the human embryo and fetus in the sense that extrapolations are made, we must ask ourselves: 'to what extent is this justified?'.

\section{The changing concept of homology}

For many years, developmental biologists, if challenged, have sought to justify the use of animal model systems by virtue of homology of form. This is likely to have been 
based loosely upon the a priori argument that, at least in early development, phenotypic similarities between human and non-human vertebrate species must reflect equivalence of the underlying generative mechanism. However, only slowly has evidence for such assumptions about homology of mechanism begun to accumulate. Perhaps one of the clearest early demonstrations of this relates to the zone of polarizing activity (ZPA) - the region of a limb bud which, by release of a diffusible morphogen, polarizes the distal part of the growing limb and controls the anteroposterior pattern of digits (see Chapter 7). It was found that the ZPA taken from a human limb bud will, when assayed by grafting ectopically into a chick embryo wing bud, display the same activity as the equivalent region of a chick bud. Extra digits are formed in a predictable and organized fashion by the host, demonstrating that human and chicken ZPAs produce the same morphogen, but chicken host cells respond to it by forming additional chick digits (Fallon and Crosby, 1977). In other words, there is an equivalence of mechanism in the building of this particular bit of anatomy. Although this example deals with just a small part of the body plan (digit specification), it can be seen as exemplifying a widely held belief that similar equivalences exist at the mechanistic level in the building of much of the anatomy or, at least, that portion of it which is characteristically and uniquely 'vertebrate' in character.

This type of assumption has been cautiously held for a number of years and, in a rather piecemeal and limited fashion, evidence gradually accumulated to give it some justification. However, it has become clear in the last few years that the concept of homology is underpinned by an amazing degree of conservation of both gene sequence and function (reviewed by Scott, 2000). So fundamental is this to our understanding of the genotype-phenotype relationship and to our interpretation of data from model systems, that it is necessary to deal with the topic at some length.

The existence of Drosophila mutants in which body parts are transformed into recognizable structures but develop at an inappropriate site, the so-called homeotic mutants, has been known since the nineteenth century, when the phenomenon of homeosis was first discovered. Certain unidentified genes were thought to be involved in the specification of the segmented body plan of Drosophila, with mutation resulting in mis-specification of particular body parts. Cloning and sequencing revealed that the homeotic genes are in fact regulatory genes and contain a highly conserved motif, the homeobox (McGinnis et al., 1984), encoding a DNA-binding domain that subsequently became known as the homeodomain. Further analysis of homeobox-containing genes confirmed their role in morphogenetic specification and revealed a complex and hierarchical genetic control of the body plan in this arthropod (reviewed by Akam et al., 1994). The cloning of these genes provided probes with which to screen the genomes of other species, and screening revealed a surprising degree of conservation, with orthologous genes being found in a very wide and diverse range of species examined. The largest and best known of these homeobox-containing gene families are the Hox genes, of which there are 39, organized in four clusters on different chromosomes in all vertebrates including humans. 
Sequence homology and position within each cluster is such that derivation of each gene can be traced from a single ancestral cluster similar to the HOM-C complex in Drosophila. Excellent reviews of the organization, evolution and functional roles of Hox genes have been published elsewhere (McGinnis and Krumlauf, 1992; Burke, 2000; Garcia-Fernàndez, 2005) and accounts of their role in specification of major features of the vertebrate body plan are given here in Chapters 7, 11, 12 and 15 .

Although these genes and others like them have only been identified in vertebrate genomes by virtue of their sequence homology with their Drosophila counterparts (remember that Drosophila probes were used in the screening), conservation of gene sequence is only one aspect of this remarkable evolutionary story. If there is truly homology of function, then we might expect conservation of expression domains of the gene(s) in question across a range of species, and this is indeed often found. The most rigorous test, however, has to be an operational one in which genes are moved into the genome of another and distant species, preferably into individuals in which the orthologue has been inactivated. Will the introduced 'foreign' gene be switched on in the correct spatiotemporal pattern and will it function to produce a normal embryo?

Homeobox-containing genes provide a number of examples in which these three criteria of sequence homology, equivalence of expression domain and functional homology are satisfied. Thus, a regulatory sequence of the Drosophila homeotic gene, Deformed, which supports expression in subregions of posterior head segments, can be replaced by the equivalent mouse sequence and still result in normal embryonic development (Awgulewitsch and Jacobs, 1992). The mouse gene, Hoxb-6, can be moved into the Drosophila embryo and specify normal thoracic segments (Malicki et al., 1990) and even the regulatory element of a human Hox gene, HOXB4, is expressed rostrally and supports head development when introduced into Drosophila (Malicki et al., 1992). Finally, we should not assume that such exchanges only operate between species with segmented body plans, no matter how divergent they may be, since it has also been shown that equivalent functional homology exists between the Hox genes of Drosophila and those of the unsegmented nematode worm, Caenorhabditis elegans (Hunter and Kenyon, 1995).

The existence of such amazing functional homology might suggest that there has been some conservation of downstream target genes for the homeoproteins. But how would this degree of conservation of homeobox gene function across a wide range of species correlate with the diverse range of phenotypic form displayed by these species? In other words, how do we reconcile functional homology, and all that that entails, with the evolution of the disparate body plans displayed by mammals and insects, for example? Such questions are currently unresolved but various possibilities, such as homeoproteins acquiring new targets, homeobox genes changing expression domains, changes in the function of downstream target genes and the emergence of new modes of regulation, are all under consideration (Kenyon, 1994; Manak and Scott, 1994; Hughes and Kaufman, 2002). Meanwhile, similar levels of conservation for genes involved in major morphogenetic events are being discovered, with functional homology apparently being retained by other key regulatory genes and 
pathways, such as goosecoid, Brachyury and non-canonical Wnt signalling controlling the very different modes of gastrulation across species as diverse as zebrafish, Xenopus, chick and mouse (Beddington and Smith, 1993; De Robertis et al., 1994; Tada et al., 2002).

However, it is not just regulatory genes that display such conservation of sequence, expression domain and function. It is rapidly emerging that genes encoding a number of secreted molecules involved in signalling between cells have been similarly conserved. Genes homologous to the Drosophila hedgehog gene family (so named because of the 'spiny' appearance of the mutant larvae) encode secreted proteins that appear to have a pivotal role in patterning a number of structures in vertebrates (reviewed by Hammerschmidt et al., 1997; Nybakken and Perrimon, 2002). The product of sonic hedgehog (shh) has a major role in notochord induction of the ventral floor plate of the neural tube (e.g. Roelink et al., 1994; and see Chapter 8). A parallel signalling role for this secreted protein is seen in limb development. Thus, shh is expressed in the posterior region of both fin (zebrafish) and limb buds (chick and mouse) where it is thought to be active in establishing pattern across the anteroposterior axis of the bud and is a component of the ZPA (see earlier). Ectopic expression of this gene in the anterior part of the chick limb bud produces duplication of anterior structures, paralleling the mirror-image duplication of the anterior wing compartment in Drosophila resulting from ectopic hedgehog expression (Fietz et al., 1994). Functional homology is even maintained amongst some of the other signalling molecules thought to be downstream from the hedgehog proteins, such as decapentaplegic $(d p p)$ in Drosophila, and the related transforming growth factor- $\beta$ (TGF- $\beta$ ) gene family in vertebrates (reviewed by Hogan et al., 1994), and the proteins with which they interact during specification of dorsoventral pattern in the neural primordium (Holley et al., 1995).

Another example of evolutionary conservation of function has recently been demonstrated with the signalling pathway for planar (epithelial) cell polarity. Epithelial cell orientation and cross-talk to the surrounding cells is critical for correct assembly of the Drosophila compound eye and uniform positioning of hairs on the wing and thorax (Strutt, 2003). This is dictated by a secreted Wnt ligand that binds to a frizzled receptor protein complex, which then signals to the nucleus via an intracellular protein called dishevelled. A similar signalling pathway with essentially the same protein components has now been found to organize cellular convergence on the dorsal midline of the mammalian embryo in order to regulate formation of the neural tube. Disruption to any of the core protein components leads to a failure of neural tube closure, as demonstrated in mice, Xenopus and zebrafish (reviewed in Copp et al., 2003; and see Chapter 8). This pathway is also required for correct orientation of the stereociliary bundles found in the mammalian inner ear (Montcouquiol et al., 2003; Curtin et al., 2003). In contrast to neural tube development, this represents a vertebrate phenotype more closely resembling the invertebrate wing hairs.

Similarly conserved function through evolution is elegantly illustrated by the study of mutations in different orthologues of the PAX6 gene (see Chapter 9). Mutations in 
PAX6 give rise to the eye defect aniridia, while the mouse orthologue turned out to be a gene formerly known as Small eye, since a loss-of-function mutation produced a microphthalmic phenotype. Both of these genes are the functional orthologues of the Drosophila Eyeless (ey) gene (Quiring et al., 1994); ey is also involved in eye development, and a loss-of-function mutation eliminates the compound eye. As a result, Aniridia, Small eye and Eyeless are collectively regarded as Pax-6 homologues with pivotal roles in eye development, whether it be the compound eye of an arthropod or the vertebrate eye (Quiring et al., 1994). This has been assessed by ectopic expression of the ey gene, which results in ectopic compound eyes with relatively normal facet organization and arrays of photoreceptor cells (Figure 1.4a). More relevant to this discussion is the finding that ectopic expression of the mouse Pax-6/Small eye gene introduced into Drosophila will also generate ectopic compound eyes that are morphologically equivalent to the normal compound eye (Figure 1.4b; Halder et al., 1995). In other words, the generative programme for assembling an arthropod compound eye can be activated and controlled by a mouse Pax- 6 gene. It is concluded that these various $\mathrm{Pax}-6$ homologues constitute master genes, arising from a common ancestral gene and with conserved function in controlling eye morphogenesis.

With the advent of more efficient positional cloning strategies, mouse knockout technology and the development of large scale ENU mutagenesis programmes, more and more genes are being assigned to function and phenotype. As a consequence, the extent of regulatory gene involvement in birth defects is becoming better defined. There are now numerous examples of regulatory gene families that are grouped
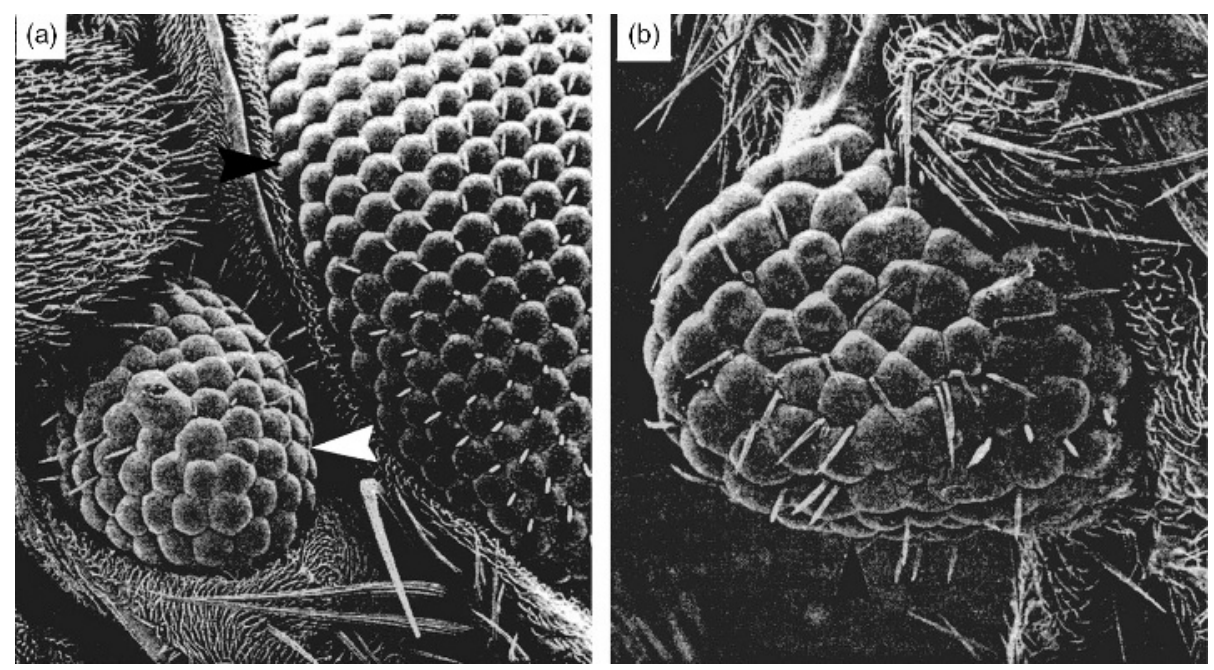

Figure 1.4 (a) Ectopic compound eye (white arrowhead) formed adjacent to the normally located compound eye (on the right, black arrowhead) in the head of a Drosophila fly; this is the result of the ectopic expression of the ey gene. (b) Ectopic compound eye formed, in this case, on the leg of a fly, under the control of an ectopically expressed mouse Pax-6 gene introduced experimentally. In both (a) and (b), note the similarity of the ommatidial organization and interommatidial bristles, in the ectopic eyes and in their normal counterpart in (a). Photographs supplied by Professor Walter Gehring 
through both sequence and functional homology that are also directly implicated in dysmorphogenesis and neoplasia. These include the PAX, HOX, ZIC, ZNF, SOX, FOX and TBX families. When the first T-box gene, $T$ (Brachyury), was identified (Herrmann et al., 1990) it was thought to be unique. However, most species studied have multiple family members and mammals contain a total of 17 different functional $T$-like genes (see Table 1.1). Family members are based on their

Table 1.1 The mammalian T-box gene family, with mouse and human mutant phenotypes

\begin{tabular}{|c|c|c|c|}
\hline Gene & $\begin{array}{l}\text { Location } \\
\text { (human) }\end{array}$ & Human phenotype & Mouse phenotype \\
\hline$T$ & $6 \mathrm{q} 27$ & $\begin{array}{l}\text { Possible risk factor } \\
\text { for NTD }\end{array}$ & $\begin{array}{l}\text { Lack notocord and } \\
\text { posterior somites }\end{array}$ \\
\hline$T b x 1$ & $22 \mathrm{q} 11$ & DiGeorge syndrome & DiGeorge syndrome-like \\
\hline$T b \times 2$ & $17 q 23$ & $\begin{array}{l}\text { Potential oncogene } \\
\text { implicated in breast } \\
\text { cancer }\end{array}$ & $\begin{array}{l}\text { Atrioventricular canal and } \\
\text { septation of the outflow } \\
\text { tract; hindlimb digits }\end{array}$ \\
\hline$T b \times 3$ & $12 \mathrm{q} 24$ & $\begin{array}{l}\text { Ulnar-mammary } \\
\text { syndrome }\end{array}$ & $\begin{array}{l}\text { Mammary gland, limb and } \\
\text { yolk sac defects }\end{array}$ \\
\hline$T b x 4$ & $17 q 23$ & Small patella syndrome & $\begin{array}{l}\text { Hindlimb bud outgrowth } \\
\text { failure }\end{array}$ \\
\hline$T b x 5$ & $12 \mathrm{q} 24$ & Holt-Oram syndrome & $\begin{array}{l}\text { Cardiac defects and } \\
\text { forelimb malformations }\end{array}$ \\
\hline$T b x 6$ & $16 \mathrm{p} 11$ & & $\begin{array}{l}\text { Abnormal patterning and } \\
\text { specification of the cervical } \\
\text { somites posterior paraxial } \\
\text { mesoderm }\end{array}$ \\
\hline Tbx 10 & $11 q 13$ & & Cleft lip and palate* \\
\hline$T b \times 15$ & $1 \mathrm{p} 12$ & & $\begin{array}{c}\text { Coat pigmentation anomalies } \\
\text { and skeletal development }\end{array}$ \\
\hline$T b x 18$ & $6 \mathrm{q} 14$ & & $\begin{array}{l}\text { Somite compartment } \\
\text { boundary formation }\end{array}$ \\
\hline$T b \times 19$ & $1 \mathrm{q} 24$ & ACTH deficiency & ACTH deficiency \\
\hline$T b \times 20$ & $7 \mathrm{p} 14$ & & $\begin{array}{l}\text { Defects in cardiac chamber } \\
\text { differentiation }\end{array}$ \\
\hline$T b \times 21$ & $17 q 21$ & $\begin{array}{l}\text { Physiological and inflammatory } \\
\text { features of ashma }\end{array}$ & \\
\hline$T b \times 22$ & $\mathrm{Xq} 21$ & X-linked cleft palate & \\
\hline Tbr1 & $2 \mathrm{q} 24$ & & $\begin{array}{l}\text { Defects in neuronal } \\
\text { migrations and axonal } \\
\text { projection }\end{array}$ \\
\hline Eomes & $3 \mathrm{p} 24$ & & $\begin{array}{l}\text { Failure of trophoblast } \\
\text { differentiation and } \\
\text { mesoderm formation }\end{array}$ \\
\hline$M G A$ & $15 q 15$ & & Unknown \\
\hline
\end{tabular}

In addition, an intronless pseudogene (TBX23) is present on chromosome 1 and a truncated T-box gene similar to TBX20 is present on chromosome 12 .

${ }^{*}$ Gain of function. 
conservation of a 180-200 amino acid sequence that encodes a DNA-binding domain, the so-called ' $\mathrm{T}$ domain' or 'T-box'. These genes function as transcription factors and are found throughout metazoans, including primitive species such as Caenorhabditis elegans and hydra. Most of the T-box genes are expressed early in development, regulating cell fate and behaviour, thereby specifying regional tissue characteristics. For example, Tbx4 and Tbx5 play major roles in limb identity (see Chapter 7). While the genes are specifically expressed in hindlimb and forelimb, respectively, ectopic expression of either has the ability to at least partially reprogramme cell fate decisions into the opposite limb type (Rodriguez-Esteban et al. 1999; Takeuchi et al. 1999). Most of the human phenotypes resulting from various TBX gene mutations occur as a consequence of haploinsuficiency, indicating that the tissues involved are especially sensitive to expression levels. It is also interesting to note that significant overlap is found in the expression domains of a number of the T-box genes (see Chapter 9). This may simply be a legacy of their ancestral origin, where expanding genome size through rounds of duplication has retained the same regulatory elements that have not yet re-specified. Nevertheless, this co-expression may allow for additional protein-protein interactions, such as heterodimerization, which in turn lead to increased complexity of function.

As yet, the biochemical pathways that are regulated by T-box genes remain poorly understood. Nevertheless, there is an increasing list of developmental defects and phenotypes being associated with the various family members (see Table 1.1). Of course, from a birth defects point of view, this is only going to be the tip of the iceberg because of the numerous upstream and downstream genes in each pathway, amplifying the number of potential targets for mutation-induced phenotypes. However, with the use of animal models and modern expression profiling techniques, dissection of these transcription factor networks is already under way and will provide the means for further disease gene discovery.

It would seem that that long-held views on the evolution of phenotypic form are being fundamentally revised. It is not simply homology of function that has driven the development of anatomical analogues through convergent evolution. There would seem to be a basic, shared genetic 'tool-kit' for development that has been retained over many millions of years (Akam et al., 1994). The different generative programmes of development have deployed this in a multitude of ways to build different phenotypes. Not surprisingly, those phenotypes are sometimes dysmorphic, as is often the case with mutations in the genes that specify developmental processes. Earlier assumptions about the extent of homology of developmental mechanisms between human and various animal model systems have been vindicated more powerfully than could have been anticipated even a few years ago.

\section{Acknowledgement}

We are most grateful to Professor Walter Gehring, who generously provided the photographs used in Figure 1.4. 


\section{References}

Akam, M., Holland, P., Ingham, P. and Wray, G. (eds) (1994) Preface. Development (suppl: Evolution of Developmental Mechanisms).

Alberch, P. (1982) Developmental constraints in evolutionary processes. In Evolution and Development, Bonner, J.T. (ed.). Springer-Verlag: Berlin; 313-332.

Alberts, B., Johnson, A., Lewis, J. et al. (2002) Molecular Biology of the Cell, 4th edn. Garland: New York.

Awgulewitsch, A. and Jacobs, D. (1992) Deformed autoregulatory element from Drosophila functions in a conserved manner in transgenic mice. Nature 358, 341-344.

Bard, J. (1993) Embryos: Colour Atlas of Development. Wolfe: London.

Barker, D.J.P. (1995) Intrauterine programming of adult disease. Mol. Med. Today 9: 418-423.

Beddington, R.S.P. and Smith, J.C. (1993) Control of vertebrate gastrulation: inducing signals and responding genes. Curr. Opin. Genet. Dev. 3: 655-661.

Burke, A.C. (2000) Hox genes and the global patterning of the somitic mesoderm. Curr. Top. Dev. Biol. 47: 155-181.

Burn, J. and Strachan, T. (1995) Human embryo research in developmental research. Nat. Genet. 11: 3-6.

Copp, A.J. (1994) Birth defects: from molecules to mechanisms. J. R. Coll. Phys. Lond. 28: 294-300.

Copp, A.J, Greene, N.D. and Murdoch, J.N. (2003) The genetic basis of mammalian neurulation. Nat. Rev. Genet. 4: 784-793.

Curtin JA, Quint E, Tsipouri V, Arkell RM et al. (2003) Mutation of Celsr1 disrupts planar polarity of inner ear hair cells and causes severe neural tube defects in the mouse. Curr. Biol. 13: 1129-1133.

De Robertis, E.M., Fainsod, A, Gont, L.K. and Steinbesser, H. (1994) The evolution of gastrulation. Development (suppl: Evolution of Developmental Mechanisms): 117-124.

Fallon, J.F. and Crosby, G.M. (1977) Polarizing zone activity in limb buds of amniotes. In Vertebrate Limb and Somite Morphogenesis, Ede, D.A., Hinchliffe, J.R. and Balls, M. (eds). Cambridge University Press: Cambridge; 55-69.

Fietz, MJ, Concordet, J.-P., Barbosa, R. et al. (1994) The hedgehog gene family in Drosophila and vertebrate development. Development (suppl: Evolution of Developmental Mechanisms): $43-51$.

Fronhofer, H.G. and Nüsslein-Volhard, C. (1986) Organisation of anterior pattern in the Drosophila embryo by the maternal gene bicoid. Nature 324: 120-1125.

Garcia-Fernàndez J. (2005) Hox, ParaHox, ProtoHox: facts and guesses. Heredity 94: $145-152$.

Gilbert, S.F. (2003) Developmental Biology, 7th edn. Sinauer: Sunderland, MA.

Gottesman, I.I. and Hanson, D.R. (2005) Human development: biological and genetic processes. Ann. Rev. Psych. 56: 263-286.

Halder, G., Callaerts, P. and Gehring, W.J. (1995) Induction of ectopic eyes by targeted expression of the eyeless gene in Drosophila. Science 267: 1788-1792.

Hammerschmidt, M., Brook, A. and McMahon, A.P. (1997) The world according to hedgehog. Trends Genet. 13: 14-21.

Herrmann, B.G., Labeit, S., Poustka, A., King, T.R. and Lehrach, H. (1990) Cloning of the T gene required in mesoderm formation in the mouse. Nature 343: 617-622. 
Hogan, B.L.M., Blessing, M., Winnier, G.E., Suzuki, N. and Jones, C.M. (1994) Growth factors in development: the role of TGF- $\beta$ related polypeptide signalling molecules in embryogenesis. Development (suppl: Evolution of Developmental Mechanisms): 53-61.

Holley, S.A., Jackson, P.D., Sasai, Y. et al. (1995) A conserved system for dorsal-ventral patterning in insects and vertebrates involving sog and chordin. Nature 376: 249-253.

Hughes, C.L. and Kaufman, T.C. (2002) Hox genes and the evolution of the arthropod body plan. Evol. Dev. 4: 459-499.

Hunter, C.P. and Kenyon, C. (1995) Specification of anterioposterior cell fates in Caenorhabditis elegans by Drosophila Hox proteins. Nature 377: 229-232.

International Human Genome Sequencing Consortium. (2004) Finishing the euchromatic sequence of the human genome. Nature 431: 931-945.

Kenyon, C. (1994) If birds can fly, why can't we? Homeotic genes and evolution. Cell 78: $175-180$.

Larsen, W.J. (2001) Human Embryology, 3rd edn. Churchill Livingstone: Edinburgh.

Malicki, J., Schughart, K. and McGinnis, W. (1990) Mouse Hox-2.2 specifies thoracic segmental identity in Drosophila embryos and larvae. Cell 63: 961-967.

Malicki, J.LC., Cianetti, C., Peschle, C. and McGinnis, W. (1992) A human HOX4B regulatory element provides head-specific expression in Drosophila embryos. Nature 358: 345-347.

Manak, J.R. and Scott, M.P. (1994) A class act: conservation of homeodomain protein functions. Development (suppl: Evolution of Developmental Mechanisms): 61-71.

Manié, S., Santoro, M., Fusco, A. and Billaud, M. (2001) The RET receptor: function in development and dysfunction in congenital malformation Trends Genet. 17: 580-589.

Marsh, J. (ed.) (1994) Neural Tube Defects. Ciba Foundation Symposium, vol 181. Wiley: Chichester.

McGinnis, W. and Krumlauf, R. (1992) Homeobox genes and axial patterning. Cell 68: 283-302.

McGinnis, W., Garber, R.L., Wirz, J., Kuriowa, A. and Gehring, W. (1984) A homologous protein-coding sequence in Drosophila and its conservation in other metazoans. Cell 37: 403-409.

McLachlan, J.C. (1986) Self-assembly of structures resembling functional organs by pure populations of cells. Tissue Cell 18: 313-320.

Monk, M. (1994) The value of man-mouse homology in human embryology. In Early Fetal Growth and Development, Ward, R.H.T. Smith, S.K. and Donnai, D. (eds). Royal College of Obstetricians and Gynaecologists: London; 63-74.

Montcouquiol M, Rachel RA, Lanford PJ, Copeland NG et al. (2003) Identification of Vangl2 and Scrb1 as planar polarity genes in mammals. Nature 423: 173-177.

Nybakken, K. and Perrimon, N. (2002) Hedgehog signal transduction: recent findings. Curr. Opin. Genet. Dev. 12: 503-511.

Opitz, J. (1985) The developmental field concept. Am. J. Med. Genet. 21: 1-11.

Polifka, J.E. and Friedman, J.M. (2002) Medical genetics: 1. Clinical teratology in the age of genomics. Canad. Med. Assoc. J. 167: 265-273.

Quiring, R., Walldorf, U., Kloter, U. and Gehring, W.J. (1994) Homology of the eyeless gene of Drosophila to the Small eye gene in mice and Aniridia in humans. Science 265: 785-789.

Reed, G.B., Rajan, K.T., Ballard, P.L., Shephard, T.H. and Wong, L. (1995) The uses of human embryonic and fetal tissues in treatment and research. In Diseases of the Fetus and Newborn, 2nd edn, Reed, G.B., Claireaux, A.E. and Cockburn, F. (eds). Chapman \& Hall: London; 389-397. 
Rodriguez-Esteban, C., Tsukui, T., Yonei, S., Magallon, J. et al. (1999) The T-box genes Tbx4 and Tbx5 regulate limb outgrowth and identity. Nature 398: 814-818.

Roelink, H., Augsburger, A., Heemskerk, J. et al. (1994) Floor plate and motor neuron induction by vhh-1, a vertebrate homologue of hedgehog expressed by the notochord. Cell 76: $7611-7775$.

Scott, M.P. (2000) The natural history of genes. Cell 100: 27-40.

Struhl, G., Struhl, K. and Macdonald, P.M. (1989) The gradient morphogen bicoid is a concentration dependent transcriptional activator. Cell 57: 1259-1273.

Strutt, D. (2003) Frizzled signalling and cell polarization in Drosophila eye. Development 130: $4501-4513$.

Sykes, B. (1993) Introduction to medical genetics. In Connective Tissue and Its Heritable Disorders. Wiley-Liss: New York; 7-50.

Tada, M., Concha, M.L., and Heisenberg, C.P. (2002). Non-canonical Wnt signalling and regulation of gastrulation movements. Semin. Cell Dev. Biol. 13: 251-260.

Takeuchi, J.K., Koshiba-Takeuchi, K., Matsumoto, K., Vogel-Hopker, A. et al. (1999) Tbx5 and Tbx4 genes determine the wing/leg identity of limb buds. Nature 398: 810-814.

Warburton, D. and Fraser, C. (1964) Spontaneous abortion risks in man: data from reproductive histories collected in a medical genetics unit. Am. J. Hum. Genet. 16: 1-15.

Wolpert, L. (1991) The Triumph of the Embryo. Oxford University Press: Oxford.

Wolpert, L. (2002) Principles of Development. Oxford University Press: Oxford. 